\title{
Intra-group relatedness affects parental and helper investment rules in offspring care
}

\author{
James L. Savage ${ }^{1 *}$, Andrew F. Russell ${ }^{2}$, Rufus A. Johnstone ${ }^{1}$ \\ ${ }^{1}$ Department of Zoology, University of Cambridge, Downing Street, Cambridge CB2 3EJ, U.K. \\ ${ }^{2}$ Centre for Ecology and Conservation, College of Life and Environmental Sciences, University of Exeter \\ Cornwall Campus, Treliever Road, Penryn TR10 9EZ, UK. \\ * Corresponding author. Email: james.savage@cantab.net
}

Published in: Behavioral Ecology and Sociobiology 67(11), 750-758.

Link:

\begin{abstract}
http://ink.springer.com/article/10.1007\%2Fs00265-013-1595-5
\end{abstract}
\begin{abstract}
In any system where multiple individuals jointly contribute to rearing offspring, conflict is expected to arise over the relative contributions of each carer. Existing theoretical work on the conflict over care has: (a) rarely considered the influence of tactical investment during offspring production on later contributions to offspring rearing; (b) concentrated mainly on biparental care, rather than cooperatively caring groups comprising both parents and helpers; and (c) typically ignored relatedness between carers as a potential influence on investment behavior. We use a game-theoretical approach to explore the effects of female production tactics and differing group relatedness structures on the expected rearing investment contributed by breeding females, breeding males, and helpers in cooperative groups. Our results suggest that the breeding female should pay higher costs overall when helpful helpers are present, as she produces additional offspring to take advantage of the available care. We find that helpers related to offspring through the breeding female rather than the breeding male should contribute less to care, and decrease their contribution as group size increases, because the female refrains from producing additional offspring to exploit them. Finally, within-group variation in helper relatedness also affects individual helper investment rules by inflating the differences between the contributions to care of dissimilar helpers. Our findings underline the importance of considering maternal investment decisions during offspring production to understand investment across the entire breeding attempt, and provide empirically testable predictions concerning the interplay between maternal, paternal and helper investment and how these are modified by different relatedness structures.
\end{abstract}

Keywords: biparental care; cooperative breeding; game theory; load-lightening; maternal effects; provisioning rules 


\section{Introduction}

In many animals, the successful development of offspring requires resources from parents beyond those delivered pre-hatching/prenatally. Offspring receiving such resources can obtain them from one parent only (uniparental care), both parents (biparental care) or at least one parent and non-parent (cooperative care). Understanding the patterns of resource investment in developing young by parents and non-parents is a central component of life-history theory (Williams 1966; Stearns 1992). Despite this, few attempts have been made to model the investment rules of carers across different stages within a breeding attempt (see Shen et al. 2011 for a two-carer example), even though changes to investment levels at an early stage in a breeding attempt are known theoretically and empirically to influence later behavior of all carers (Smith and Härdling 2000; Russell et al. 2007) as well as estimations of carer investment rules (Russell et al. 2008). In addition, few studies have explored how the indirect fitness consequences of relatedness among cares should influence investment in a cooperative group (Johnstone 2011).

In uniparental care systems, the predicted maternal investment strategy within a given reproductive attempt is relatively straightforward, as it is only influenced by the relationships between current investment, and both current and future contributions to overall fitness (Williams 1966). When another carer is added, contributions to care becomes more complex because the optimal investment strategy of one parent is also influenced by that of the other, as each will benefit when the other takes on a greater proportion of the costs of care (Trivers 1974). Assuming carers have similar information about offspring need (Johnstone and Hinde 2006), the usual prediction of biparental care models is that parental investment should be reduced to incompletely compensate for that provided by the other partner (Houston and Davies 1985; McNamara et al. 1999); although threshold effects (Jones et al. 2002) or very high responsiveness to partners (McNamara et al. 2003) can lead to other response rules. Cooperative care systems further complicate the possible investment strategies, not only because the parent(s) and non-parent(s) may accrue different benefits when contributing to offspring care (Hatchwell 2009), but because both the number of non-parents (hereafter helpers) and their relatedness to each other and to offspring can vary both within and between species (Russell and Lummaa 2009). This complexity may explain why empirical tests of provisioning rules are largely supportive of incomplete compensation in biparental species (Harrison et al. 2009), whilst cooperative species have been found to exhibit almost every conceivable response to the presence of other carers (Hatchwell 1999; Heinsohn 2004). To date, few theoretical frameworks have been provided in which to understand this variation.

Using a comparative approach, Hatchwell (1999) found that in cooperatively breeding birds a significant proportion of the striking variation in investment rules could be explained by the probability of offspring starvation. In bird species in which nestling starvation was rare, parents are more likely to compensate for 
additional investment from others, while in species with frequent nestling starvation, additive care is more common (where each additional carer increases the overall investment to the brood). In a three-player negotiation model, Johnstone (2011) showed that this result could be accounted for by different rearing cost functions: where such functions are more strongly accelerating (i.e. conditions are harsher and offspring payoffs lower), all carers tend towards maintaining investment levels such that offspring receive the largest possible amount of resources. In addition, Johnstone (2011) suggested an important role for intra-group relatedness in determining response rules: when rearing cost functions are similar for both parents, the presence of a helper leads to the parent that is less related to the helper compensating for changes in helper effort more strongly. However, both the above studies have important limitations.

Firstly, breeding females vary dramatically in their initial investment in offspring production, both within and between species (Russell and Lummaa 2009), and this investment is likely to significantly influence maternal care allocations during offspring rearing, and by extension the contributions of other carers (Clutton-Brock et al. 2004; Gilchrist and Russell 2007). Previous studies have rarely considered a role of maternal investment during offspring production on carer investment optima during offspring rearing, and those that have (e.g. Shen et al. 2011) seldom gave control of offspring number to the breeding female. However, growing evidence suggests that breeding females are capable of modifying the number and/or quality of offspring produced when they can predict the likely contributions of other carers (Davies et al. 1992; Russell et al. 2003; Woxvold and Magrath 2005; Russell et al. 2007; Taborsky et al. 2007; Canestrari et al. 2011; Santos and Macedo 2011; Paquet et al. 2013). In a recent game-theoretic model, Savage et al. (2013) showed that high production costs (relative to rearing costs) should lead to reductions in individual investment with additional carers, because in such cases breeding females produce fewer offspring, which consequently need less investment during the rearing period. However, this model was limited by treating the breeding male as identical to other helpers, and by neglecting to explore the effects of intra-group relatedness on allocations of care.

Secondly, while Johnstone (2011) considered relatedness structure between the breeding pair and a single helper, the potential effects of having multiple helpers of varying relatedness were not explored. It is rarely the case that cooperatively breeding groups are restricted to parents with a single helper, yet few attempts have been made to predict how individuals will respond to each other in larger, interrelated cooperative groups: at most, three individuals have been considered (Härdling et al. 2003; Johnstone 2011). The relatedness between helpers and breeders within cooperative groups can vary considerably both between and within species, because although cooperative breeding typically arises within a family context (Emlen 1995; Hatchwell 2009), helpers may be related to both parents, one parent (after the other dies/disperses or following group immigration) or neither parent (when both are replaced from outside the group or when the 
helper is a lone immigrant) (Stacey and Koenig 1990; Dickinson and Hatchwell 2004). Variation in kinstructure is likely to influence helper provisioning rules (and by association the parental rules) because related helpers will gain a greater benefit from reducing the costs of each other's contributions than will unrelated helpers, either because related helpers that survive to become breeders will provide each other with inclusive fitness, or because related helpers are more likely to agree on an appropriate level of investment to deliver to future broods of offspring (West-Eberhard 1975).

For the present study we define a cooperative breeding system as any in which non-breeding helpers contribute to rearing the offspring of others. In addition, for the purposes of our model, we assume that all helpers behave similarly with no significant task specialization or qualitative differences in investment strategy, as is commonly the case in cooperative breeders (Clutton-Brock et al. 2003; Cockburn 2004; Russell 2004). We present a game-theoretical model of a cooperative breeding system in which the breeding female can choose the number of offspring she produces with knowledge of the number of helpers that will assist in rearing them. We then explore the effects of varying the number of helpers and the relatedness differences among breeding females, breeding males, helpers and offspring on: (i) the expected payoffs; (ii) provisioning rules; and (iii) individual levels of investment in the system. We show that several different results should be expected for the same level of intra-group relatedness, depending on the number of carers contributing, but that higher intra-group relatedness should largely lead to reductions in individual investment by all carers when additional carers are added to a given system.

\section{Methods}

We model the breeding attempt as a two-step sequential game, following Smith and Härdling (2000) and Savage et al. (2013). In the first step, the breeding female chooses the number of offspring to produce, with knowledge of the number of carers that will contribute to the breeding attempt, and pays a personal cost for producing each offspring. Following this, the entire group plays a "sealed bid" investment game (Houston and Davies 1985) to determine the investment levels of each individual contributing to care. The sealed-bid approach simplifies the analysis considerably over "negotiation" approaches (as in McNamara et al. 1999, 2003; Johnstone and Hinde 2006; Johnstone 2011), and the predictions of the two modeling frameworks are qualitatively similar, particularly when individual responsiveness to other carers is low or moderate (McNamara et al. 2003). We use the same cost and benefit functions as Savage et al. (2013) (see below), but rather than focusing on the effects of costs incurred at different stages of reproduction (production and rearing), we instead explore the effects of relatedness structure on individual contributions to care. We consider three different classes of carer: the breeding female, the breeding male, and a variable number of 
helpers. The helpers may be related to the breeding female, the breeding male, or both, and we also explore cases in which two different types of helper contribute to care within the same breeding attempt.

We model the cooperative breeding group as an interrelated set of individuals comprising two parents (breeding female and breeding male) and $\mathrm{H}$ helpers (focusing on $0 \leq H \leq 10$, which encapsulates the majority of mean group sizes across cooperative vertebrates; Russell and Lummaa 2009). The breeding female and breeding male are assumed to be unrelated to each other, and each related to their offspring by $r=0.5$. Helpers are assumed to be related to the offspring and to each other ( $r_{h o}$ and $r_{h h}$ respectively) by values between 0 and 0.5 . Helpers can be related to offspring through either the breeding female, breeding male, or both, and so female-helper and male-helper relatedness ( $r_{f h}$ and $r_{m h}$ respectively) are also assumed to vary between 0 and 0.5 . In natural systems the paired relatedness values between an interrelated group of helpers, offspring, and breeders will never be fully independent, so in several cases we alter $r_{h o}, r_{f h}, r_{m h}$ and $r_{h h}$ together as a single variable termed "group relatedness" $\left(r_{g}\right)$ to focus are results on realistic parameter's. In other cases we hold one variable constant and explore the effects of varying another, for example when quantifying the differences between half-sibling helpers $\left(r_{h o}=0.25\right)$ related through each parent $\left(0 \leq r_{f h} \leq 0.5\right)$.

We assume that all group members are able to respond to the number of offspring produced by the female in the first (offspring production) step by adjusting their level of investment in the second (offspring rearing) step. We treat all helpers of the same type (e.g. female-related half-siblings) as identical, ignoring potential consequences of variation among helpers in ability to deliver care. To find stable levels of investment for all individuals, we first calculate the response of the breeding female, both types of helper and breeding male to a given brood size $n$. The breeding female chooses a level of investment $x_{f}$, and simultaneously the breeding male and helpers choose a level of investment, designated $x_{m}$ for the male and $x_{h}^{i}$ for the $i^{\text {th }}$ helper. We assume that offspring benefit from investment through an increase in survival probability and future reproductive opportunities, and that total investment is divided equally among all offspring. As such, the benefit $b$ to offspring depends only on $n$ and the total investment all individuals provide, $x_{\text {sum }}$, where $x_{\text {sum }}=x_{f}+x_{m}+\sum_{i=1}^{H} x_{h}^{i}$. To more realistically approximate the development of offspring we define a minimum level of investment per offspring $x_{\text {min }}$ below which offspring survival is not possible and hence carers gain no benefit from investment. Consequently, $b$ responds to investment as a function of $\left(x_{\text {sum }} / n\right)-x_{\text {min }}$. We assume that the first derivative of the benefit function must be positive, such that greater investment leads to greater benefit to the offspring, but that the second derivative must be negative, such that there are diminishing returns on increasing investment. To obtain precise results we use a simple illustrative function that satisfies these assumptions (over the appropriate range of values) and yields an analytical solution:

$b=k_{b} \cdot\left(\left(\frac{x_{\text {sum }}}{n}-x_{\min }\right)-\left(\frac{x_{\text {sum }}}{n}-x_{\min }\right)^{2}\right)$ 
Individuals pay a cost $c$ for investing in offspring, determined entirely by their own individual level of investment. We define the total cost to the female $c_{f}$ as a function of both the number of offspring produced by the female $n$, and her level of investment in rearing the brood $x_{f}$. To account for variability in the costliness of producing and rearing offspring, the relative costs of offspring production and rearing are manipulated by defining two constants $k_{p}$ and $k_{r}$, which act on $n$ and $x_{f}$ respectively. The final cost $c_{f}$ to the female is the sum of the costs incurred over the two steps, as in natural systems both production and rearing of offspring is costly and often necessitates a trade-off by females (Monaghan et al. 1998). We assume that the first derivative of the cost function $\left(c^{\prime}\right)$ is positive for all individuals, such that costs increase with greater investment, and that the second derivative $\left(c^{\prime \prime}\right)$ is also positive, such that costs accelerate as investment increases. As before, a simple quadratic function is used as an illustrative example:

$c_{f}=\left(k_{p} \cdot n^{2}+k_{r} \cdot x_{f}{ }^{2}\right)$

Similarly, for the $i^{t h}$ helper the cost $c_{i}^{h}$ depends on the helper's investment level $x_{h}^{i}$ and the relative cost of offspring rearing (a new constant $k_{s}$, to allow for differences from the female's rearing costs). We also define the cost $c_{m}$ for the male in terms of his investment $x_{m}$ and a constant $k_{m}$. Unlike the female's cost function neither the female nor the male's cost functions depend on $n$, as the other carers do not produce offspring themselves.

$c_{i}^{h}=\left(k_{s} \cdot x_{h}^{i 2}\right)$

$c_{m}=\left(k_{m} \cdot x_{m}^{2}\right)$

We assume that each individual chooses its level of investment so as to maximize a weighted sum $(w)$ of the benefit to the offspring minus its personal costs and the costs incurred by the other carers in the group, where the weighting of each term corresponds to the relatedness to the focal individual of the individual incurring the cost or benefit. At this point differences in relatedness values between different classes of helper become important, so we define two helper classes $h_{l}$ and $h_{2}$, with $H_{A}$ and $H_{B}$ helpers in each class $\left(H=H_{A}+H_{B}\right)$, and allow their investment $x_{h}^{i}$ to differ $\left(x_{h 1}^{i}\right.$ and $\left.x_{h 2}^{j}\right)$. The relatedness values between all types of carer are assumed to be reciprocal, and are shortened to the form $r_{x y}$, where $x$ and $y$ are abbreviations of the focal carer types (e.g. $r_{f h l}$ for the relatedness value between females and class 1 helpers), or the form $r_{x o}$ for the relatedness between carer type $x$ and the offspring. Breeding females and breeding males are assumed to be unrelated. To summarize, the maximands (respectively $w_{f}, w_{m}, w_{h 1}^{i}$ and $w_{h 2}^{j}$ for the $i^{t h}$ helper of class $h_{1}$ and $j^{\text {th }}$ of class $h_{2}$ ) are given by: 


$$
\begin{aligned}
& w_{f}=r_{f o} \cdot b-c_{f}-r_{f h 1} \cdot \sum_{i=1}^{H_{A}} c_{h}^{i}\left(x_{h 1}^{i}\right)-r_{f h 2} \cdot \sum_{j=1}^{H_{B}} c_{h}^{j}\left(x_{h 2}^{j}\right) \\
& w_{m}=r_{m o} \cdot b-c_{m}-r_{m h 1} \cdot \sum_{i=1}^{H_{A}} c_{h}^{i}\left(x_{h 1}^{i}\right)-r_{m h 2} \cdot \sum_{j=1}^{H_{B}} c_{h}^{j}\left(x_{h 2}^{j}\right) \\
& w_{h 1}^{i}=r_{h 10} \cdot b-c_{h}^{i}-r_{f h 1} \cdot c_{f}-r_{m h 1} \cdot c_{m}-r_{h 1 h 1} \cdot \sum_{i=1}^{H_{A}-1} c_{h}^{i}\left(x_{h 1}^{i}\right)-r_{h 1 h 2} \cdot \sum_{j=1}^{H_{B}} c_{h}^{j}\left(x_{h 2}^{j}\right) \\
& w_{h 2}^{j}=r_{h 2 o} \cdot b-c_{h}^{j}-r_{f h 2} \cdot c_{f}-r_{m h 2} \cdot c_{m}-r_{h 2 h 2} \cdot \sum_{j=1}^{H_{B}-1} c_{h}^{j}\left(x_{h 2}^{j}\right)-r_{h 2 h 1} \cdot \sum_{j=1}^{H_{A}} c_{h}^{i}\left(x_{h 1}^{i}\right)
\end{aligned}
$$

At equilibrium no individual can increase their payoff by altering their investment levels, i.e.:

$$
\frac{\partial w_{f}}{\partial x_{f}}=\frac{\partial w_{m}}{\partial x_{m}}=\frac{\partial w_{h 1}^{i}}{\partial x_{h 1}^{i}}=\frac{\partial w_{h 2}^{j}}{\partial x_{h 2}^{j}}=0
$$

Solving the above produces rules specifying $x_{f}, x_{m}, x_{h 1}^{i}$ and $x_{h 2}^{j}$ ). in terms of $n$ and $H$ at equilibrium, which are termed, $\hat{x}_{f}, \hat{x}_{m} \quad \hat{x}_{h 1}^{i}$ and $\hat{x}_{h 2}^{j}$ respectively. Within each helper class there are no intrinsic differences between the helpers in our model, so each should make the same response to investment by the rest of the group, and hence at equilibrium $x_{h 1}^{i}$ and $x_{h 2}^{j}$ are each identical for all $i$ or for all $j$.

We are now able to calculate the optimal number of offspring for the breeding female to produce in the first step of the model, taking into account the impact of her choice on the subsequent investment decisions of all group members. The female should choose a value of $n$ such that it maximizes $w_{f}$, given that $x_{f}=\hat{x}_{f}(n)$, $x_{m}=\hat{x}_{m}(n), x_{h 1}^{i}=\hat{x}_{h 1}^{i}(n)$ and $x_{h 2}^{j}=\hat{x}_{h 2}^{j}(n)$ at equilibrium. For simplicity, we treat $n$ as a continuous variable (a reasonable approximation when $n$ is large). Formally, at equilibrium:

$$
\frac{\partial w_{f}}{\partial n}=0
$$

This leads to equilibrium rules for $x_{f}, x_{m}, x_{h 1}^{i}, x_{h 2}^{j}$ and $n$ at equilibrium that depend only on helper number $\mathrm{H}$ and the extrinsically specified cost parameters $\left(k_{b}, k_{p}, k_{r}, k_{s}, k_{t}\right)$. All solutions were generated and subsequent plots produced in Wolfram Mathematica (v7.01, Wolfram Research).

To plot results we chose values for the constants such that investment levels ranged between 0 and 1 , and such that numbers of offspring approximated a natural range for a terrestrial vertebrate. This leads to a relationship of approximately $k_{r}=20 k_{p}$. We also assume that all individuals have the same cost constants through the rearing stage $\left(k_{r}=k_{s}=k_{t}\right.$ ), such that everyone pays the same costs for delivering the same level of investment to the brood (see Savage et al. 2013) for an exploration of differences in this parameter between helpers and females, and for the effects of varying the $k_{r}: k_{p}$ ratio). 


\section{Results}

\section{(a) Offspring production}

Our model predicts that patterns of relatedness within groups should have a strong influence on the number of offspring the breeding female produces. Breeding females should always increase investment in offspring production as the number of available helpers increases, but the magnitude of this increase is determined by both the female's relatedness to the helpers $\left(r_{f h}\right)$ and the helpers' relatedness to the offspring $\left(r_{h o}\right)$. Malehelper $\left(r_{m h}\right)$ and helper-helper $\left(r_{h h}\right)$ relatedness values have no effect on the equilibrium levels of investment by the female, male or helpers, and hence no effect on the investment per offspring delivered by the group. As cooperative groups often consist of individuals who are interrelated to some degree (aside from the breeding pair), in some cases we assume that $r_{f h}$ and $r_{h o}$ vary together as a single parameter (group relatedness, $\left.r_{g}\right)$, Under this condition, females in highly interrelated groups $\left(r_{g}=0.5\right)$ show greater investment in offspring production than those in moderately $\left(r_{g}=0.25\right)$ or distantly interrelated groups $\left(r_{g}=0.125\right)$ (Fig. 1a). This effect largely arises because helpers that are closely related to offspring are willing to invest more during the rearing period, and the female is able to produce more offspring accordingly.

When $r_{f h}$ and $r_{h o}$ are instead altered independently (i.e. when comparing male-related helpers to femalerelated helpers), female investment behavior is less straightforward because the two parameters affect offspring production in opposite directions. Greater helper-offspring relatedness encourages greater investment by the female (as above) because the helpers will contribute more to care, but females also reduce investment in offspring production as female-helper relatedness increases. Breeding females invest more in offspring production when they are less closely related to helpers (assuming $r_{h o}$ is constant) because they benefit from reducing the costs borne by related individuals during the breeding attempt (Fig. 1b). In essence, the benefit the female receives from rearing greater numbers of offspring is lessened by the impact on the costs and survival probability of related helpers. Consequently, a breeding female should invest maximally in offspring production when the other carers are closely related to the offspring but distantly related to her, as she has no negative fitness consequences from extracting more investment from them. The breeding male represents a class of carer that fulfills the above conditions for maximal female investment (high relatedness to offspring, zero to female), and as such his presence or absence has a greater effect on the female's tactics during offspring production than does that of any single helper: all helpers that are related to offspring as closely as the breeding male are also offspring of the breeding female (Fig. 1c). 
$1 \mathrm{a}$

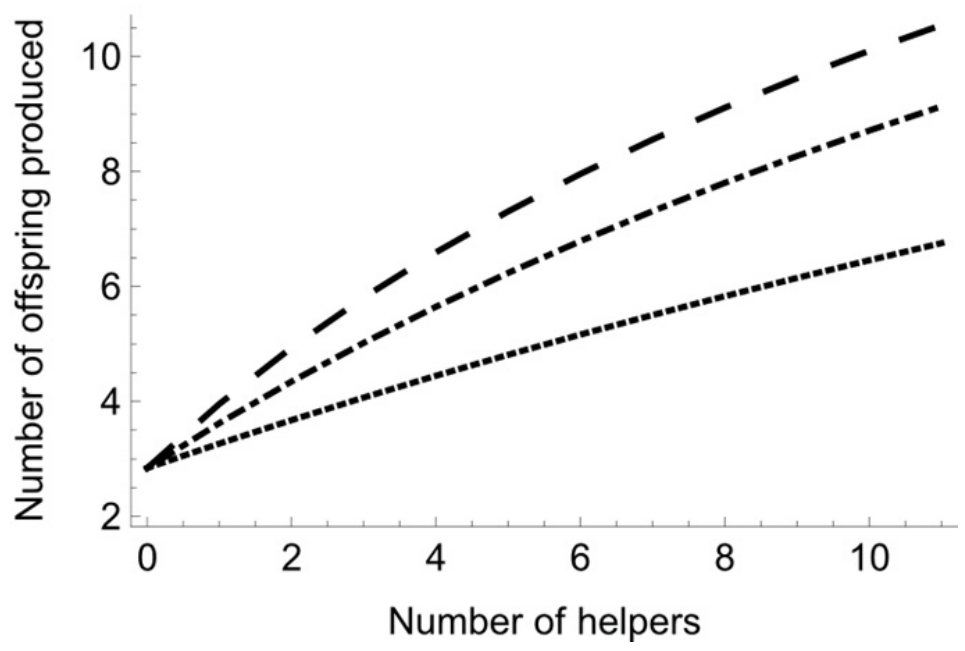

$1 b$

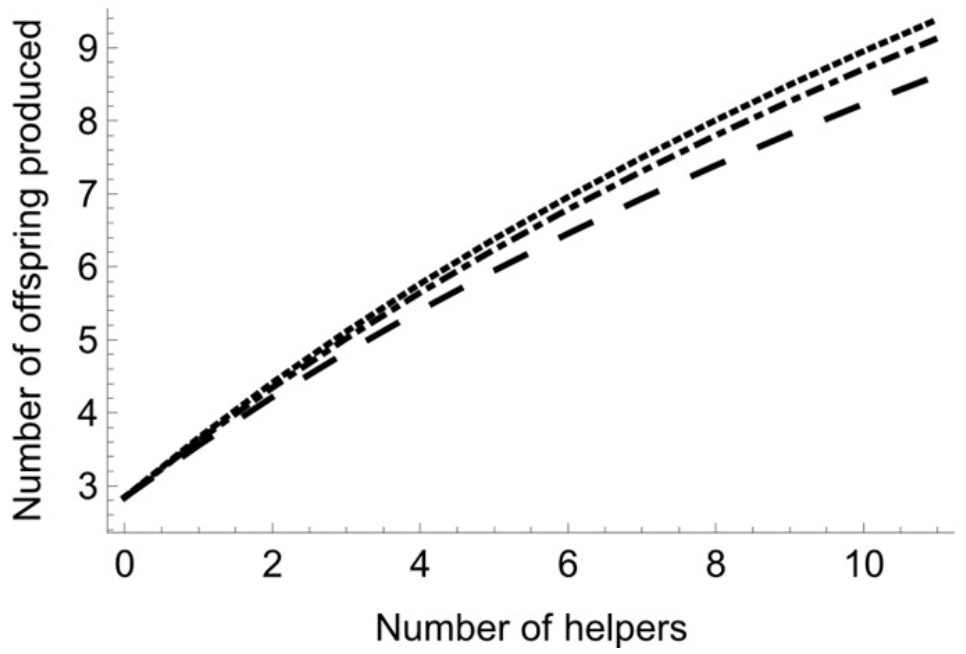

$1 \mathrm{c}$

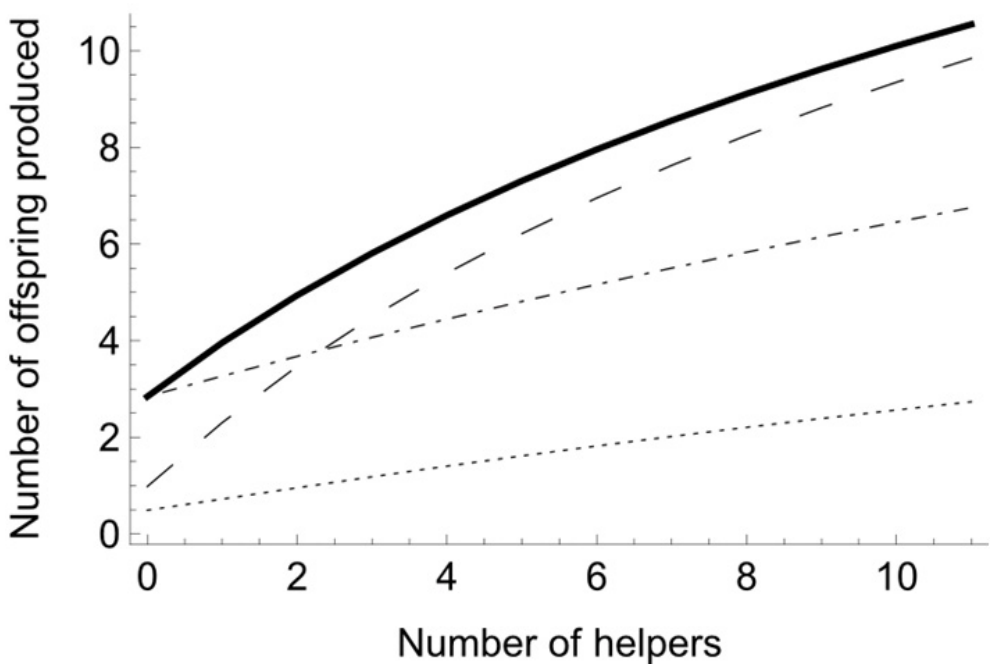

Fig. 1: (a) Females produce more offspring with more helpers, but are expected to respond to closely related helpers by increasing offspring production further. The plot shows offspring production for close $\left(r_{g}=0.5\right.$, dashed), intermediate $\left(r_{g}=0.25\right.$, dotdashed), or distant $\left(r_{g}=0.125\right.$, dotted $)$ relatedness between group members. (b) When only relatedness between females and helpers is considered, close relatedness leads to lower investment by females at the rearing stage due to costs being shared. In the graph, helperoffspring relatedness is fixed at 0.25 and offspring production is plotted for different levels of female-helper relatedness $\left(r_{f h}=0.5\right.$ (dashed), 0.25 (dotdashed), 0.125 (dotted)). (c) A male is both closely related to offspring and distantly related to the female, and therefore exerts a stronger influence on female production than does a single helper. Lines show offspring production in groups with a male and helpful $\left(r_{h o}\right.$ $=0.5$ ) helpers (solid), with a male and unhelpful $\left(r_{h o}=0.125\right)$ helpers (dotdashed), with helpful helpers but no male (dashed), and with unhelpful helpers with no male (dotted) 
$2 \mathrm{a}$

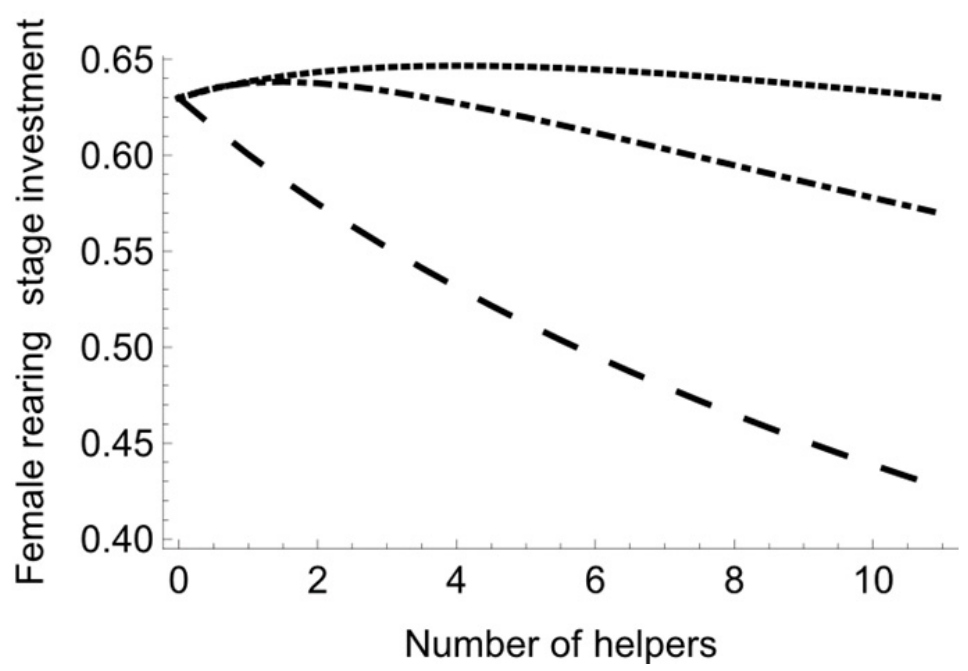

$2 b$

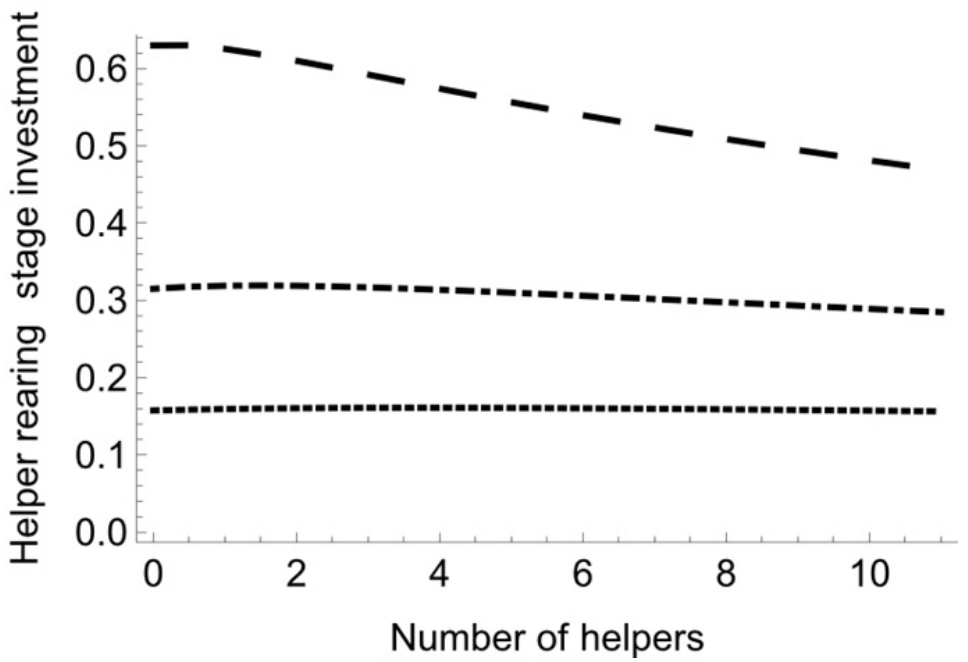

$2 \mathrm{c}$

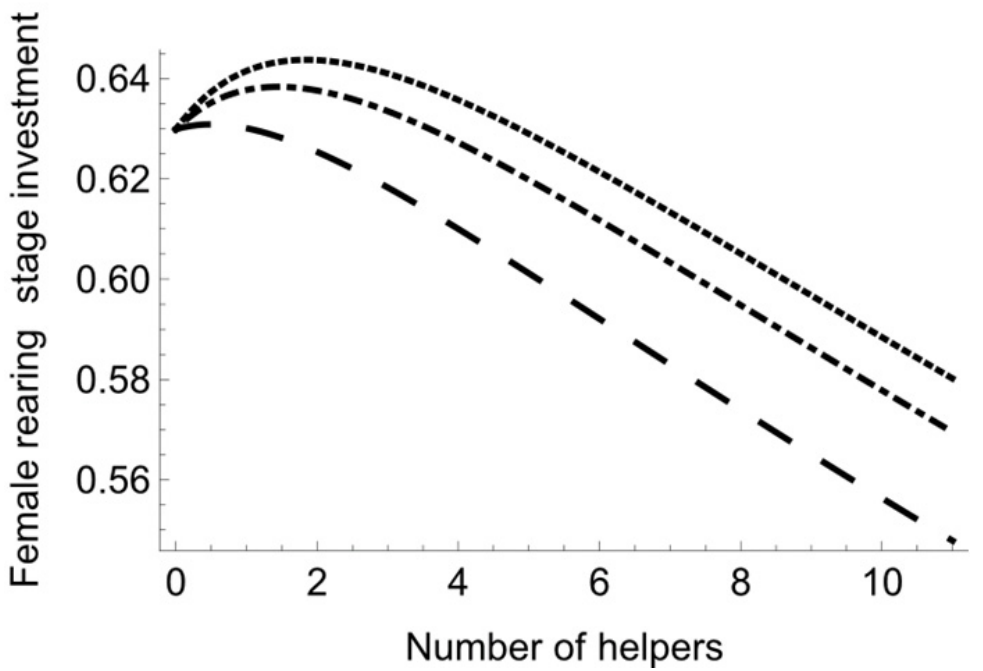

Fig. 2: (a) Females reduce rearing-stage investment when they have helpful helpers (high group relatedness), as they benefit more by overproducing in the first step due to the greater available amount of care. (b) Helpers invest more in closely related offspring, and exhibit a greater reduction in individual contribution at high helper numbers. Plots show helper investment for close ( $r_{g}=0.5$, dashed), intermediate $\left(r_{g}=0.25\right.$, dot-dashed $)$, or distant $\quad\left(r_{g}=0.125\right.$, dotted) relatedness between group members. (c) When only relatedness between females and helpers varies, there is a reduction in care by females when helpers are related to her, as she benefits less from extracting more investment from them. Helper-offspring relatedness is fixed at $r_{h o}=0.25$ and the female rearing-stage investment plotted for different levels of female-helper relatedness: $\left(r_{f h}=0.5\right.$ (dashed), 0.25 (dot-dashed), 0.125 (dotted)) 


\section{(b) Offspring rearing}

Variation in offspring production by the breeding female under differing patterns of relatedness and different numbers of helpers has knock-on effects on the investment by all carers during offspring rearing. As above, we initially assume that $r_{f h}$ and $r_{h o}$ vary together as the single parameter "group relatedness" $\left(r_{g}\right)$. When group relatedness and helper number increase, the female increases offspring production to take advantage of the available care, but eventually the personal costs she incurs from doing so prohibit further increases. At this point the only effect of contributions from extra helpers is to reduce costs to each other and the costs borne by the breeding female, and the benefits individuals derive from doing this will depend on the level of group relatedness. In closely-related groups, compensatory care with additional helpers is predicted across all group sizes, as the female reaches her constraints on offspring production more quickly with harder-working helpers, and all individuals benefit from reducing the costs paid by each other. In less closely-related groups, no compensation for extra helpers is seen at first, as the female modifies her investment in offspring production in response to the number of carers, but it eventually appears when sufficient helpers are present (Fig. 2a).

Unsurprisingly there is also a positive effect of group relatedness on helper investment levels, with close relatives $\left(r_{g}=0.5\right)$ investing more than those of intermediate relatedness $(0.25)$ and those in turn investing more than distant relatives (0.125). Helpers also reduce individual investment when there are many helpers and group relatedness is high, but maintain largely static investment levels across differing group sizes when group relatedness is low (Fig. 2b). As before, when female-helper relatedness is allowed to vary independently of helper-offspring relatedness, the effect of higher $r_{f h}$ is to lower the investment by the female (Fig.and 2c). This arises because of the lower rearing investment required to support the reduced number of offspring produced in the first step, and as with offspring production suggests that female-unrelated helpers should behave more like the breeding male than female-related helpers, and have a more similar effect to on group investment behavior.

\section{(c) Between- and within-group variation in relatedness}

In natural systems helpers may be related to offspring through the breeding female or breeding male, and because of the fitness dependency between related carers this distinction is important even when helperoffspring relatedness is identical. Comparing groups in which all helpers are similar, full-sibling helpers invest more than half-sibling helpers due to greater benefits, but among half-sibling helpers those that are breeding-female-related invest less than those that are breeding-male-related, because the female produces less offspring in the first step when helpers related to her are present (Fig. 3a). Full-sib helpers reduce investment as group size increases in order to escape the costs of care, because the female is already at or 
close to her limit of offspring production. In contrast half-sib helpers largely maintain investment levels over a range of group sizes because the female can still invest more in offspring production in response to extra helpers.

A similar pattern can be observed when comparing the outcomes for individual offspring across group relatedness structures (Fig. 3b). Investment-per-offspring increases with helper number when helpers are closely related to the female, but does not when helpers are unrelated to her and group size is small. This is because the female produces fewer offspring in total when she has a small number of related helpers than when she has a small number of unrelated helpers, so the total investment has to be divided into fewer portions by the offspring. With the female being more willing to exploit unrelated than related helpers, each additional unrelated helper leads to a large increase in offspring production, and although all carers increase their investment levels to provide for these additional offspring, this increase may not fully compensate for the greater total investment required; this leads to each offspring receiving less care. In contrast, when a related helper is added to a group the female makes a more modest increase in offspring production, and consequently the presence of an extra carer leads to total investment by the group outpacing the number of offspring, and each individual offspring receiving more care.

When helpers vary in relatedness within a group, each helper still responds independently to the number of offspring produced as a function of its own costs and its relatedness to the offspring and other carers, and the female tunes offspring production to the sum of potential investment across all the helpers. Consequently we find that investment in a mixed-relatedness helper group should be equivalent to investment in a homogenous helper group that possesses the same relatedness values between all carers and offspring as the mean values of the mixed group. However, it is instructive to compare the investment by a particular type of helper within a homogenous group of its own helper type with the investment by the same helper in a mixed group in which other helper types are also present. In these cases mean relatedness values to the female and offspring will vary between the groups compared, so the female strategy and investment levels of all carers will differ. We find that full-sib helpers invest more in mixed groups where half-sib helpers are also present than in homogenous groups where all helpers are full-sibs, as there is less investment by the rest of the helper group. Conversely, half-sib helpers invest less in a mixed group as the female is less willing to exploit them by producing additional offspring when there are full-sib helpers present that will provide the majority of the care (Fig. 3c). As a consequence, between-group differences in relatedness have less influence on individual investment levels than within-group differences: full-sib and half-sib helpers differ in investment level more when both are present in the same group than in groups with only one type of helper. 


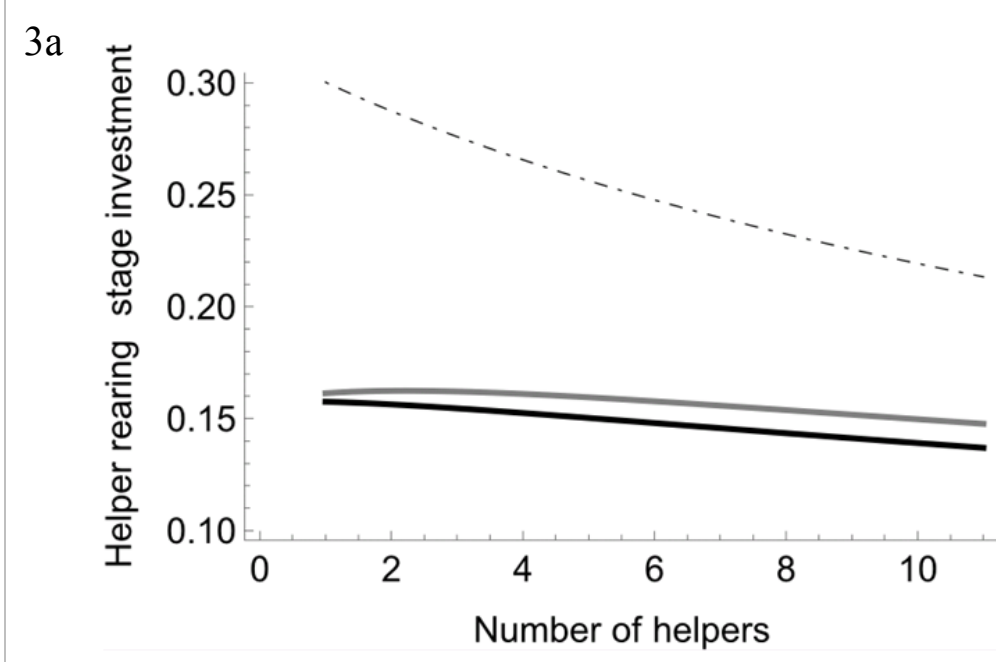

$3 b$

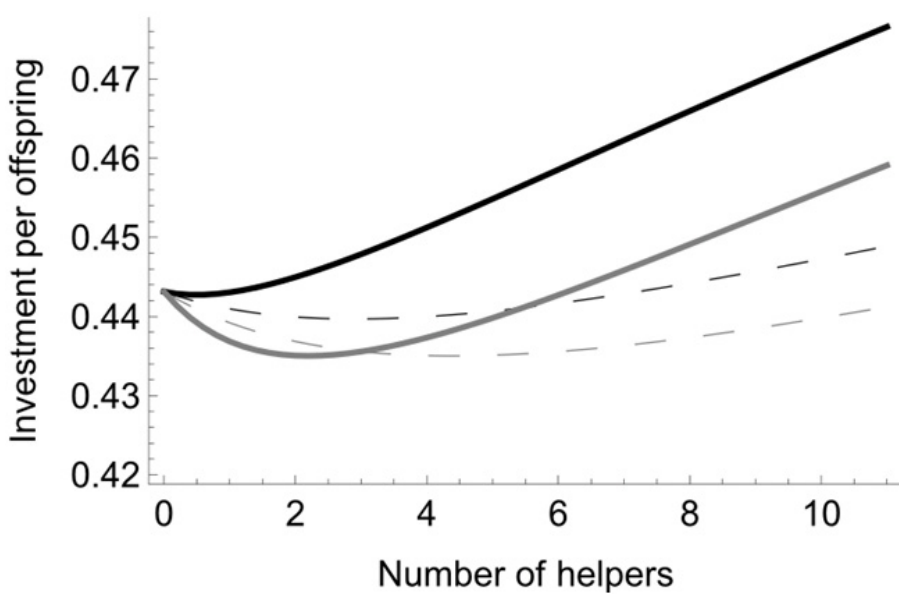

$3 c$

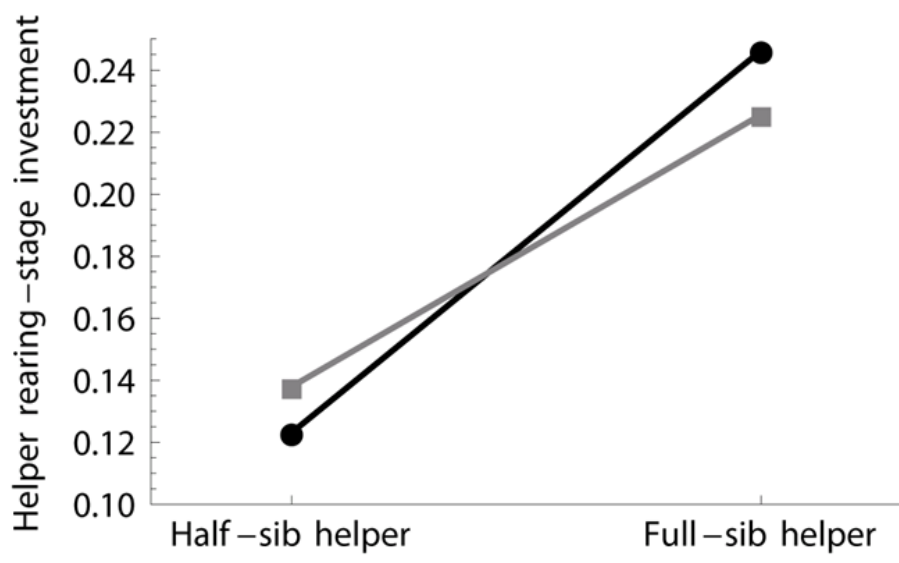

Fig. 3: (a) Helper investment in groups where helpers are full-siblings to offspring is greater than in groups where helpers are halfsiblings, and when group size increases individual reductions in care are more pronounced. In addition, because of a reduction in production-stage investment by the breeding female, helpers invest less in groups where they are closely related to the female. (b) Individual offspring benefit from additional helpers that are closely related to the breeding female, particularly when both $r_{f h}$ and $r_{h c}$ (i.e. $r_{g}$ ) are high. When $r_{f h}$ is low, offspring may do less well individually as group size increases, because the female produces more offspring in total to exploit the helpers. Plots show investment-peroffspring for groups with half-sib helpers (solid lines) and distantly-related $\left(r_{f o}=0.125\right)$ helpers (dashed lines) that are either closely related $\left(r_{f h}=0.5\right.$, black), or unrelated $\left(r_{f h}=0\right.$, grey) to the breeding female. The black dotdashed line represents the investment level of a full-sib helper related to both parents and offspring by 0.5 . (c) Within-group variation in relatedness has a greater influence on helper investment levels than does betweengroup variation. Half-sib helpers invest less in mixed groups than in homogenous groups in which all helpers are half-sibs, whereas full-sib helpers invest more in mixed groups than in homogenous groups in which all helpers are full-sibs. The plot shows helper investment levels for full-sib and half-sib helpers in mixed groups (black, 3 full-sib and 3 male-related half-sib helpers), and homogenous groups (grey, 6 helpers that are either full-sibs or male-related half-sibs) 


\section{Discussion}

Although there is accumulating evidence that females tactically adjust their level of investment according to socio-ecological conditions, relatively little theoretical work exists on the subject. As such, our work extends previous models in two key ways. Firstly, it allows for flexible maternal investment in offspring as a function of the number and relatedness of available carers, treating helpers and the breeding male separately. Secondly, it explores the effects of variable helper numbers and different relatedness levels on carer investment patterns during offspring rearing. Our results suggest that intra-group relatedness will have a significant influence on the provisioning rules and levels of investment observed in cooperative breeding systems. Broadly, female productivity increases as a function of increasing intra-group relatedness due to the increased benefits to helpers of investing heavily in more related offspring. However, the effect of helpers on female productivity was found to be lower than the effect of the breeding male, because, in contrast to related helpers, the breeding female has no indirect fitness stake in his survival. In addition, the circumstances under which carers reduce investment when more helpers are present are strongly influenced by intra-group relatedness, as extracting investment from related individuals is less beneficial. Our results have implications for provisioning rules on multiple levels, from differences between biparental, polyandrous, and cooperative care systems, to the influences of dispersal strategy and breeding tenure on group provisioning and investment over time (see Fig. 4).

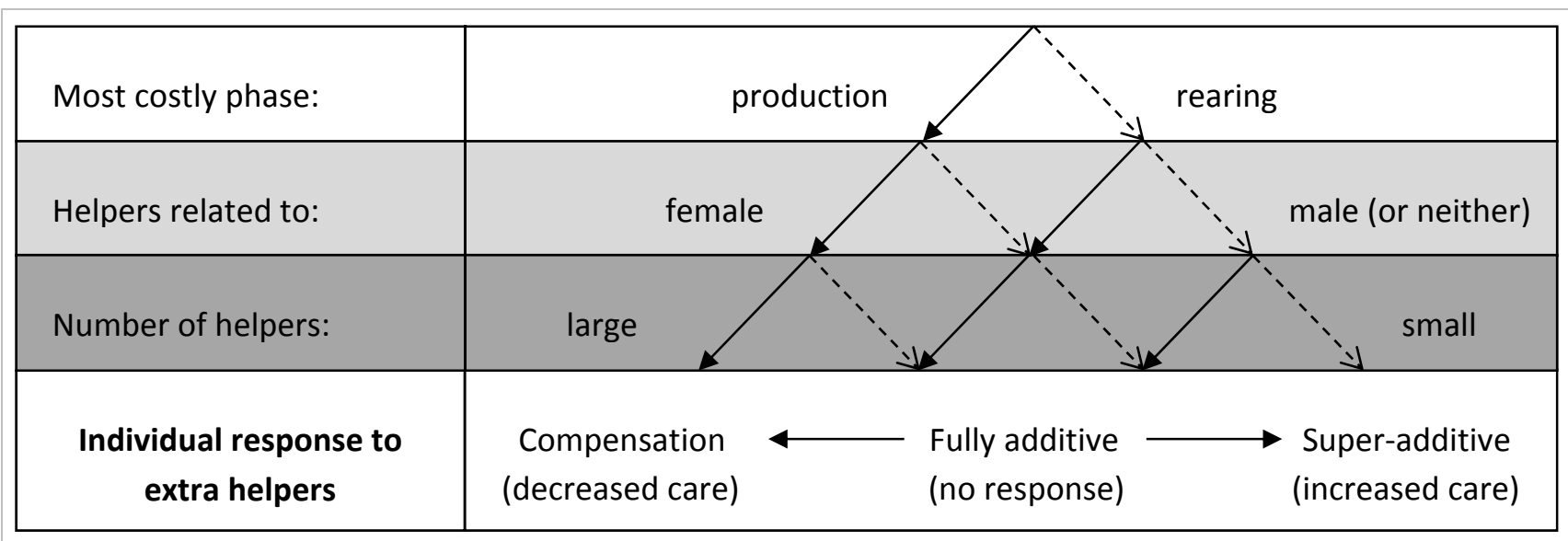

Fig. 4: Predicted investment rules across different helper numbers within cooperative groups, based on the distribution of costs to the breeding female, the relatedness of helpers to the breeding female and the current number of helpers. Reduction of individual carer investment as helper number increases (i.e. compensation or "load-lightening") is expected when (1) offspring production is relatively more costly to the female than offspring rearing (see Savage et al. 2013), (2) when the breeding female is closely related to the helpers (or directly benefits from their survival), and (3) when the current number of helpers is high. By contrast, helpers are expected to maintain (leading to additive care) or in extreme cases increase (leading to super-additive care) investment levels when production costs are low, helpers are unrelated to the female and the number of helpers is low. These results arise from the female tactically increasing offspring number by varying amounts to take advantage of the extra available care 
The effects of maternal tactics on individual provisioning rules are particularly clear when our results are compared with previous theoretical work modeling investment in offspring during a single reproductive stage. In contrast to a female with (up to) two helpers rearing young (Härdling et al. 2003), we do not always see a reduction in individual carer investment as group size increases: if the female can increase her production of offspring, carers may maintain or even increase their investment. In addition, in our model total female costs are higher (rather than lower) with full-sib helpers, as the female increases offspring number to take account of the greater potential help. Our model contrasts similarly with previous work on a breeding pair and single helper rearing young (Johnstone 2011), as we find that carers reduce their individual investment less when the female has tactical control of offspring number. However we support Johnstone's (2011) finding that females will pay lower costs with related helpers than with helpers related only to their partner provided that the helpers' relatedness to the offspring is the same. Modeling investment as a twostage process and with a range of group sizes shows that carer investment strategies can strongly deviate from those predicted by analyses of the rearing stage in isolation, particularly when group size also varies, and can additionally be modified by the relatedness structure of the group (see Russell et al. 2008 for some supporting empirical evidence).

Our prediction that individuals should provision differently according to relatedness patterns in the group has consequences for comparing provisioning rules in cooperative breeding systems to those in biparental systems. Biparental care is well-analyzed, and whether sealed-bids (Houston and Davies 1985), negotiation (McNamara et al. 1999; Johnstone and Hinde 2006; Johnstone 2011), or sequential negotiation (Lessells \& McNamara 2012) are used to determine investment levels, the results generally predict partial compensation in the presence of a partner (though see McNamara et al. 2003; Johnstone and Hinde 2006). Cooperative systems (when considered theoretically at all) have often been regarded as direct extensions of the biparental case, with simply more individuals that invest in offspring according to their own costs in the same way as the parents; only rarely has theoretical work explicitly addressed investment consequences of variation in inter-carer relatedness (Härdling et al. 2003; Johnstone 2011). However, under our model, a biparental system differs significantly from a cooperative one in investment behavior, as biparental systems contain only unrelated carers while cooperative ones usually display some degree of interrelatedness. In particular, the relatedness between the carers modifies how much females are willing to exploit the other carers by modifying their own investment across offspring production and rearing. As a consequence, we predict that provisioning rules in biparental systems will differ from those in cooperative systems when indirect fitness relationships exist among carers, for example by the breeding female exhibiting weaker responses to the presence/absence of another carer in cooperative systems. 
Intra-group variation in relatedness patterns in cooperative breeders might help to explain the significant variation in observed carer provisioning rules. While investigations into parental provisioning rules in biparental care systems tend to provide support for expected incomplete compensation (Harrison et al. 2009), this is less commonly the case in cooperative breeders (Hatchwell 1999; Heinsohn 2004). For example, of the 27 cooperative bird species in Hatchwell's (1999) comparative analysis on provisioning rules, helpers were associated with reductions in provisioning rate of both parents (44\%), one parent only (30\%) or neither parent $(26 \%)$. This variation was shown to be attributable to variation in nestling starvation: carers tend to reduce investment when carer numbers increase if nestling starvation is rare, but maintain investment where nestling starvation is common (Hatchwell 1999). However, all else being equal, our model also suggests that individual reductions in rearing-stage investment when there are more carers ("load-lightening") should be a common empirical finding over a wide range group sizes when females and helpers in a group are closely interrelated. By contrast, when females and helpers are more distantly interrelated, their investment levels should remain largely constant regardless of group size because females alter offspring production to extract the maximum possible investment from all carers. Consequently, our model could provide part of the mechanism behind Hatchwell's (1999) findings: the observed inter-species differences in nestling starvation may arise in part from the consequences of variable maternal investment in offspring production, group size, group kin-structure, and the interplay between them.

That said, an emerging pattern in many cooperatively breeding vertebrates is that different categories of carers react differently to the presence of co-carers. For example, in meerkats (Suricata suricatta) breeders of both sexes reduce their investment in pup-feeding with increasing group size, while non-breeders maintain their levels (Clutton-Brock et al. 2004). Such results are not consistent with the above hypothesis regarding offspring starvation risk (Hatchwell 1999), and have instead been interpreted as breeders benefiting more than helpers from saving resources for future reproduction, particularly in species where the probability of attaining breeder status is low (Clutton-Brock et al. 2004; Browning et al. 2012). Our results suggest that an additional explanation might be the costs of offspring production and relatedness differences between carers in the group. In particular, females constrained from or selected against producing more offspring when helper number increases are expected to reduce their investment, while helpers investing at lower levels largely maintain their contributions to better support the offspring. If enough helpers are available at the rearing stage, every group member benefits from a greater proportion of the female's investment being made at the initial stage. In groups with helpers closely related to the breeding female, one would expect greater reductions in investment as helper number increases, as a consequence of the female being unwilling to foist additional costs on to the helpers by increasing offspring production. Studies have yet to consider how carers of differing relatedness to each other should respond to changes in group size, but our model predicts several possible effects, including differences in investment in mixed group relative to homogenous groups, and a 
greater similarity of investment behavior between the breeding male and helpers related to offspring through him, compared to those related through the breeding female.

While some caution is required due to the lack of demographic and ecological considerations in our model, our results also have implications for how female investment should vary across species with the system of dispersal and length of breeding tenure. When females are unrelated to helpers but helpers still contribute heavily to care we predict an exploitative strategy with females producing more offspring to extract more investment from the other carers. In contrast, a female with closely related helpers should produce fewer offspring, causing an overall reduction in the costs all carers pay. Given that the former case will usually arise when females are dispersive and the latter when females are philopatric, we predict that in general birds should respond more strongly than mammals to changes in helper numbers by altering offspring numbers, except in species with sex-biased dispersal opposite to the norm (Greenwood 1980). Exploitative strategies should be most prominent in newly immigrated breeding females, because through their breeding tenure they will become increasingly related to their helpers (Johnstone \& Cant 2010). Mathematical models and empirical work show that such effects could have significant consequences on reproductive skew in humans (Cant \& Johnstone 2008; Lahdenperä et al. 2012), but the consequences for cooperative breeding animals remain untested. Although potentially difficult to implement, a demographically explicit version of our model could provide more complex predictions about investment behavior, as helpers might (for example) be expected to adjust their contributions to care based on their own sex, the sex of the offspring, and the sex bias in dispersal, because of the potential for kin competition (Johnstone \& Cant 2008). Similarly, we have ignored variation in the costs of cooperation when compared to alternative options for helpers, which is likely to modify the degree to which females can extract additional investment from helpers as group size changes.

Finally, despite our framing of the model in terms of indirect fitness benefits, the link between helpers and offspring in our analysis has implications for any scenario in which helpers have an interest in the survival of the offspring and other group members. For example, under group augmentation (Kokko et al. 2001), helpers benefit by successfully raising additional offspring to boost their own direct fitness, and hence within a single breeding attempt the benefits from helping are similar to those generated by inclusive fitness relationships. We suggest that our model is a fair approximation for this type of direct fitness benefit, particularly for smaller values of intra-group "relatedness", which would be more accurately termed fitness interdependence in the case of directly benefiting helpers (see Roberts 2005). The analogy also holds for between-carer inclusive fitness relationships: individuals concerned with augmenting group size will be averse to other carers paying high costs, as raising additional group members is of limited benefit if current group members perish in the process. We would expect carers benefiting through group augmentation to 
maintain investment patterns through time regardless of changes in breeder identity, with the benefit attached to group augmentation replacing "group relatedness", i.e. the breeding female should reduce her contribution at the rearing stage when augmentation benefits are high, focusing instead on producing additional offspring. Given the current lack of empirical research on the topic, we suggest that future work on cooperative care systems should investigate how provisioning rules are influenced by variation in the benefits of group size for different classes of individual.

To summarize, maternal control of offspring production and inter-carer relatedness and can both have strong influences on the expected provisioning rules in biparental and cooperative systems. Such rules include the numbers of offspring produced, the response to additional carers, and quantitative investment levels during provisioning; and we can make empirical predictions about how the rules might be expected to vary within and between species (Fig. 4). Our results underline the importance of incorporating tactical female investment when modeling the conflict over care: when a female is able to predict the number and helpfulness of other carers in advance of producing offspring, her ability to manipulate the initial investment can lead to different behaviors among other members of the group than if her investment were fixed. Consequently individual investment decisions cannot be meaningfully analyzed in isolation, either from other carers or from the other stages in which individuals can contribute (Smith and Härdling 2000; Savage et al. 2013), and understanding the investment decisions of any group that cooperates to rear offspring ultimately depends on the integration of models of offspring number, offspring size, carer number, carer relationships, and alternative carer options. In addition, the biology of the particular species in question will affect the relative costs and constraints of producing offspring compared to rearing them (Savage et al. 2013), further complicating the issue by altering the proportion of total costs helpers are able to contribute to. Future empirical work should monitor investment across all possible stages of a breeding attempt whenever feasible, as without wider context investment behavior can easily be misconstrued (Russell et al. 2008). Similarly, theoretical work that focuses entirely on fine-scale investment within a single stage of a breeding attempt might be expected to have limited relevance outside of its stated context.

\section{Acknowledgements}

This work was supported by a Natural Environment Research Council Studentship to the University of Cambridge (J.L.S.) and by a Royal Society University Research Fellowship (A.F.R.). We are grateful to two anonymous reviewers for their helpful suggestions. 


\section{References}

Browning, L. E., Young, C. M., Savage, J. L., Russell, D. J. F., Barclay, H., Griffith, S. C. \& Russell, A. F. 2012. Carer provisioning rules in an obligate cooperative breeder: prey type, size and delivery rate. Behavioral Ecology and Sociobiology, 66, 1639-1649.

Canestrari, D., Marcos, J. M. \& Baglione, V. 2011. Helpers at the nest compensate for reduced maternal investment in egg size in carrion crows. Journal of Evolutionary Biology, 24, 1870-1878.

Cant, M. A. \& Johnstone, R. A. 2008. Reproductive conflict and the separation of reproductive generations in humans. Proceedings of the National Academy of Sciences of the United States of America, 105, 5332-5336.

Clutton-Brock, T. H., Russell, A. F. \& Sharpe, L. L. 2003. Meerkat helpers do not specialize in particular activities. Animal Behaviour, 66, 531-540.

Clutton-Brock, T. H., Russell, A. F. \& Sharpe, L. L. 2004. Behavioural tactics of breeders in cooperative meerkats. Animal Behaviour, 68, 1029-1040.

Cockburn, A. 2004. Mating systems and sexual conflict. In: Ecology and evolution of cooperative breeding in birds, (Ed. by W. D. Koenig \& J. L. Dickinson), pp. 81-101. Cambridge University Press.

Davies, N. B., Hatchwell, B. J., Robson, T. \& Burke, T. 1992. Paternity and parental effort in dunnocks Prunella modularis: how good are male chick-feeding rules? Animal Behaviour, 43, 729-745.

Dickinson, J. L. \& Hatchwell, B. J. 2004. Fitness consequences of helping. In: Ecology and evolution of cooperative breeding in birds, (Ed. by W. D. Koenig \& J. L. Dickinson), pp. 48-66. Cambridge University Press.

Emlen, S. T. 1995. An evolutionary theory of the family. Proceedings of the National Academy of Sciences of the United States of America, 92, 8092-8099.

Gilchrist, J. S. \& Russell, A. F. 2007. Who cares? Individual contributions to pup care by breeders vs non-breeders in the cooperatively breeding banded mongoose (Mungos mungo). Behavioral Ecology and Sociobiology, 61, 10531060.

Greenwood, P. 1980. Mating systems, philopatry and dispersal in birds and mammals. Animal Behaviour, 28, 11401162.

Härdling, R., Kokko, H. \& Arnold, K. E. 2003. Dynamics of the caring family. American Naturalist, 161, 395-412.

Harrison, F., Barta, Z., Cuthill, I. C. \& Székely, T. 2009. How is sexual conflict over parental care resolved? A metaanalysis. Journal of Evolutionary Biology, 22, 1800-1812.

Hatchwell, B. J. 1999. Investment strategies of breeders in avian cooperative breeding systems. American Naturalist, 154, 205-219.

Hatchwell, B. J. 2009. The evolution of cooperative breeding in birds: kinship, dispersal and life history. Philosophical Transactions of the Royal Society B: Biological Sciences, 364, 3217-3227.

Heinsohn, R. G. 2004. Parental care, load-lightening, and costs. In: Ecology and evolution of cooperative breeding in birds, (Ed. by W. D. Koenig \& J. L. Dickinson), pp. 67-80. Cambridge University Press.

Houston, A. I. \& Davies, N. B. 1985. The evolution of cooperation and life history in the dunnock, Prunella modularis. In: Behavioural Ecology: Ecological Consequences of Adaptive Behaviour, (Ed. by R. M. Sibly \& R. H. Smith), pp. 471-487. Blackwell Scientific Publications.

Johnstone, R. A. 2011. Load lightening and negotiation over offspring care in cooperative breeders. Behavioral Ecology, 22, 436-444. 
Johnstone, R. A. \& Cant, M. A. 2008. Sex differences in dispersal and the evolution of helping and harming. American Naturalist, 172, 318-330.

Johnstone, R. A. \& Cant, M. A. 2010. The evolution of menopause in cetaceans and humans: the role of demography. Proceedings of the Royal Society B: Biological Sciences, 277, 3765-3771.

Johnstone, R. A. \& Hinde, C. A. 2006. Negotiation over offspring care-how should parents respond to each other's efforts? Behavioral Ecology, 17, 818-827.

Jones, K. M., Ruxton, G. D. \& Monaghan, P. 2002. Model parents: Is full compensation for reduced partner nest attendance compatible with stable biparental care? Behavioral Ecology, 13, 838-843.

Kokko, H., Johnstone, R. A. \& Clutton-Brock, T. H. 2001. The evolution of cooperative breeding through group augmentation. Proceedings of the Royal Society B: Biological Sciences, 268, 187-196.

Lahdenperä, M., Gillespie, D. O. S., Lummaa, V. \& Russell, A. F. 2012. Severe intergenerational reproductive conflict and the evolution of menopause. Ecology Letters, 15, 1283-1290.

Lessells, C. M. \& McNamara, J. M. 2012. Sexual conflict over parental investment in repeated bouts: negotiation reduces overall care. Proceedings of the Royal Society B: Biological Sciences, 279, 1506-1514.

McNamara, J. M., Gasson, C. E. \& Houston, A. I. 1999. Incorporating rules for responding into evolutionary games. Nature, 401, 368-371.

McNamara, J. M., Houston, A. I., Barta, Z. \& Osorno, J. L. 2003. Should young ever be better off with one parent than with two? Behavioral Ecology, 14, 301-310.

Monaghan, P., Nager, R. G. \& Houston, D. C. 1998. The price of eggs: increased investment in egg production reduces the offspring rearing capacity of parents. Proceedings of the Royal Society B: Biological Sciences, 265, 1731-1735.

Paquet, M., Covas, R., Chastel, O., Parenteau, C. \& Doutrelant, C. 2013. Maternal effects in relation to helper presence in the cooperatively breeding sociable weaver. PLOS ONE, 8, e59336.

Roberts, G. 2005. Cooperation through interdependence. Animal Behaviour, 70, 901-908.

Russell, A. F. 2004. Mammals: comparisons and contrasts. In: Ecology and evolution of cooperative breeding in birds, (Ed. by W. D. Koenig \& J. L. Dickinson), pp. 210-227. Cambridge University Press.

Russell, A. F. \& Lummaa, V. 2009. Maternal effects in cooperative breeders: from hymenopterans to humans. Philosophical Transactions of the Royal Society B: Biological Sciences, 364, 1143-1167.

Russell, A. F., Brotherton, P. N. M., McIlrath, G. M., Sharpe, L. L. \& Clutton-Brock, T. H. 2003. Breeding success in cooperative meerkats: effects of helper number and maternal state. Behavioral Ecology, 14, 486-492.

Russell, A. F., Langmore, N. E., Cockburn, A., Astheimer, L. B. \& Kilner, R. M. 2007. Reduced egg investment can conceal helper effects in cooperatively breeding birds. Science, 317, 941-944.

Russell, A. F., Langmore, N. E., Gardner, J. L. \& Kilner, R. M. 2008. Maternal investment tactics in superb fairywrens. Proceedings of the Royal Society B: Biological Sciences, 275, 29-36.

Santos, E. S. A. \& Macedo, R. H. 2011. Load lightening in southern lapwings: group-living mothers lay smaller eggs than pair-living mothers. Ethology, 117, 547-555.

Savage, J. L., Russell, A. F. \& Johnstone, R. A. 2013. Maternal costs in offspring production affect investment rules in joint rearing. Behavioral Ecology, 24, 750-758.

Shen, S.-F., Reeve, H. K. \& Vehrencamp, S. L. 2011. Parental care, cost of reproduction and reproductive skew: a general costly young model. Journal of Theoretical Biology, 284, 24-31. 
Smith, H. G. \& Härdling, R. 2000. Clutch size evolution under sexual conflict enhances the stability of mating systems. Proceedings of the Royal Society B: Biological Sciences, 267, 2163-2170.

Stacey, P. B. \& Koenig, W. D. Eds. 1990. Cooperative breeding in birds: long-term studies of ecology and behavior. Cambridge University Press.

Stearns, S. C. 1992. The Evolution of Life Histories. Oxford: Oxford University Press.

Taborsky, B., Skubic, E. \& Bruintjes, R. 2007. Mothers adjust egg size to helper number in a cooperatively breeding cichlid. Behavioral Ecology, 18, 652-657.

Trivers, R. L. 1974. Parent-offspring conflict. American Zoologist, 14, 249-264.

West-Eberhard, M. 1975. The evolution of social behavior by kin selection. Quarterly Review of Biology, 50, 1-33.

Williams, G. C. 1966. Natural selection, the costs of reproduction, and a refinement of Lack's principle. American Naturalist, 100, 687-690.

Woxvold, I. A. \& Magrath, M. J. L. 2005. Helping enhances multiple components of reproductive success in the cooperatively breeding apostlebird. Journal of Animal Ecology, 74, 1039-1050. 